PSICOLOGIA, SAÚDE \& DOENÇAS, 2015, 16(1), 3-9

EISSN - 2182-8407

Sociedade Portuguesa de Psicologia da Saúde - SPPS - www.sp-ps.com

DOI: http://dx.doi.org/10.15309/15psd160102

\title{
EDUCAÇÃO PARA A SAÚDE
}

\author{
José Luís Pais Ribeiro \\ Faculdade de Psicologia e Ciências da Educação / Universidade do Porto
}

\begin{abstract}
RESUMO -Neste artigo fazemos uma apresentação da importância e da emergência da educação para a saúde, salientando os principais marcos que lhe deram origem. Fazemos referência aos aspetos da educação como a idade, contexto e objetivos que mudam de pessoa para pessoa ou de grupo para grupo. Ilustramos com a referência a programas cuja aplicação em amplos setores, está documentada na literatura científica, justificando a sua importância e utilidade.

Palavras-chave - Educação para a saúde; promoção da saúde; prevenção das doenças
\end{abstract}

\section{HEALTH EDUCATION}

\begin{abstract}
In this article we present the importance and emergence of health education, highlighting the major milestones that gave rise to it. We refer to aspects of education such as age, context and goals that change between individuals or groups. We illustrated with reference to programs whose implementation in broad sectors are documented in the scientific literature, justifying its importance and usefulness.
\end{abstract}

Key words- health education; health promotion; disease prevention

Recebido em 20 de Maio de 2013/ Aceite em 20 de Outubro de 2014

Após a Segunda Grande Guerra Mundial, terminada em 1945, ocorreram grandes alterações na forma de conceber a saúde e as doenças. Tal decorreu, principalmente, devido ao aparecimento da penicilina que ficou disponível durante a guerra, e que permitia combater as doenças infeciosas que mais contribuíam para a mortalidade e a morbilidade da época, e devido ao desenvolvimento da vacinação que prevenia algumas das principais doenças como, p.ex., a tuberculose, entre muitas outras. A utilização de novos medicamentos e de formas de diagnóstico mais sofisticadas, levou a que a expectativa de vida tivesse aumentado substancialmente, principalmente nos países economicamente mais desenvolvidos.

Por outro lado, viver mais tempo permitiu o aparecimento de doenças não infeciosas que se tornaram, nos decénios seguintes, a principal causa de morbilidade e mortalidade nos países desenvolvidos (doenças cardiovasculares e oncológicas que representam cerca de $60 \%$ das causas de morte nestes países). Esta alteração do padrão de morbilidade e mortalidade ficou conhecida por Segunda Revolução da Saúde (PaisRibeiro, 1998) e caracteriza-se por as suas principais causas estarem associadas ao comportamento humano em vez de agentes infeciosos, como anteriormente.

\footnotetext{
Faculdade de Psicologia e Ciências da Educação / Universidade do Porto; e-mail: jlpr@ fpce.up.pt
} 
Logo após a Segunda Grande Guerra iniciou-se o Framingham Heart Study, sob os auspícios do atual National Heart, Lung, and Blood Institute, que visava identificar os fatores ou características que contribuíam para as doenças cardiovasculares e que constituiu a matriz para identificar os principais fatores de risco que hoje são do senso comum $^{1}$, e que incluem muitos dos comportamentos que atualmente são objeto de educação no âmbito da prevenção primária e da promoção e proteção da saúde. De salientar que a investigação atual tem mostrado que a relação entre fatores de risco e as doenças cardiovasculares, tal como foram identificados nos estudos originais não confirmam esse padrão (Ebrahim et al. 2011).

Este tipo de investigação que apontava para o papel do comportamento humano no aparecimento de doenças tornou a educação para a saúde um elemento central em contexto de saúde e de doenças em todos os países, desenvolvidos ou em desenvolvimento, em todos os contextos, da prevenção primária à terciária, e de promoção e proteção da saúde, em todos os grupos etários. Agora, a abordagem das doenças já não era da responsabilidade do sistema de cuidados de saúde, mas sim da responsabilidade individual.

\section{EDUCAÇÃO PARA A SAÚDE}

Educação para a saúde é definida pela Organização Mundial de Saúde (OMS) como todas e quaisquer combinações de experiências de aprendizagem concebidas para ajudar os indivíduos e as comunidades a melhorar a sua saúde, através do aumento do seu conhecimento, ou, influenciando as suas atitudes (WHO, 2013). Mais detalhadamente, a definição do glossário de promoção da saúde de 1998 (WHO, 1998), semelhante a esta, diz que educação para a saúde inclui as oportunidades desenvolvidas intencionalmente para a aprendizagem, que envolve alguma forma de comunicação, destinada a melhorar a literacia em saúde, incluindo o aumento do conhecimento, e o desenvolvimento de perícias de vida (life skills) os quais conduzem à saúde da comunidade (em itálico no original). A educação para a saúde é central seja para a promoção e proteção da saúde, seja para a prevenção das doenças (primária, secundária, terciária)

\section{Saúde}

A saúde define-se como um estado de bem-estar físico, mental e social, total, e não apenas a ausência de doença, ou de incapacidade, segundo a Organização Mundial de Saúde (OMS) no seu documento constituinte (WHO, 1948). A declaração introdutória da constituição da OMS que precedia a definição de saúde afirmava que aqueles princípios eram básicos para a felicidade, e para as relações harmoniosas e seguras de todas as pessoas: Afirmava, ainda, que a saúde de todos os povos é fundamental para alcançar a paz e segurança.

Em acrescento posterior diz que a saúde é a extensão em que um indivíduo ou grupo é capaz, por um lado, de realizar as suas aspirações e satisfazer as suas necessidades, e por outro, de modificar ou lidar com o meio envolvente. A saúde é um recurso para a vida do dia a dia, uma dimensão da nossa qualidade de vida e não o objetivo de vida, dizia (WHO, 1986).

\footnotetext{
${ }^{1}$ Ver: https://www.framinghamheartstudy.org/about-fhs/research-milestones.php
} 
Por sua vez, a saúde mental é um estado de bem-estar no qual o indivíduo implementa as suas capacidades, enfrenta o stresse normal da vida diária, trabalha produtivamente e contribui positivamente para a sua comunidade (Herrman, Saxena, \& Moodie, 2005). A saúde mental é parte integrante da saúde, é mais do que ausência de doença mental, e está intimamente conectada com a saúde física e o comportamento (Herrman, et al, 2005).

Ao contrário, a doença é a condição anormal de um corpo ou organismo, caracterizada por um conjunto de sintomas ou sinais específicos. A etiologia, patologia e prognóstico podem ser conhecidos ou desconhecidos, e ela pode ser causada por fatores externos ou internos. Ou seja, pela definição formal, saúde e doença são conceitos independentes, que podem coexistir e que não se eliminam mutuamente. Faz sentido pensar a saúde em pessoas que têm uma qualquer doença, do mesmo modo que se pode investigar doenças em pessoas dadas como saudáveis.

\section{As políticas planetárias de saúde}

$\mathrm{Na}$ década de 70 do século passado, formalizou-se em documentos clássicos, o reconhecimento da alteração das causas das doenças e da importância da saúde para a vida das pessoas e da comunidade. Salientam-se nesta época o relatório Lalonde (1974) sobre o estado de saúde dos canadianos, o relatório Richmond (1979) sobre o estado de saúde nos Estados Unidos da América, e a declaração de Alma-Ata (1978). Estes três marcos, permitem afirmar que se entrava numa nova era, quer no modo de conceber a saúde e as doenças, quer no modo de intervir.

Em 1978 é formalizada a Declaração de Alma-Ata, produzida numa conferência internacional focada nos cuidados de saúde primários, que decorreu em Alma-Ata, Cazaquistão $^{2}$, entre 6-12 Setembro. Neste documento referia-se que os cuidados primários $(\mathrm{CP})$ refletiam e derivavam das condições económicas, socioculturais, e das características políticas de cada país e das comunidades que o compunham. Afirmava que os $\mathrm{CP}$ focavam os principais problemas da comunidade, através do fornecimento de serviços promocionais, preventivos, curativos e de reabilitação. Dizia, no ponto VII da declaração que os CP incluíam, pelo menos, a educação em relação a problemas prevalecentes de saúde e a métodos para sua prevenção e controlo, promoção da distribuição de alimentos e de nutrição apropriada, provisão adequada de água de boa qualidade e saneamento básico, cuidados de saúde materna e infantil, incluindo o planeamento familiar, imunização contra as principais doenças infeciosas, prevenção e controlo de doenças endémicas, tratamento adequado de doenças e lesões comuns, e fornecimento de medicamentos essenciais.

Nesta conferência assumiu-se que o objetivo principal dos governos e da OMS seria que toda a população do planeta deveria alcançar, no ano 2000, um nível de saúde que conduzisse a uma vida social e economicamente produtiva. Esta assunção levou a que em 1981 a assembleia adotasse unanimemente a "Global Strategy for Health for All by the Year 2000", dando origem ao movimento "Saúde para Todos". Isto não significava o fim das doenças e incapacidades, mas antes que os recursos e os cuidados de saúde passavam a ser acessíveis a toda a população. Também significava que a "saúde"

\footnotetext{
${ }^{2}$ Em 1978 Alma-Ata era a capital de uma das repúblicas da União das Republicas Socialistas Soviéticas (URSS), o Cazaquistão. Posteriormente esta república tornou-se independente (1991), denominando-se República do Cazaquistão, em 1994 o nome de Alma-Ata foi substituído por Almaty continuando a ser a capital. Posteriormente a capital foi mudada para outra cidade, Astana a atual capital deste país.
} 


\section{EDUCAÇÃO PARA A SAÚDE}

começava em casa, nas escolas, no local de trabalho, na comunidade, etc., ou seja, nos contextos onde as pessoas viviam, consoante a sua idade, a sua cultura, a sua atividade, os seus interesses. Adaptado à região europeia definiam 38 objetivos para a saúde das pessoas (Ritsatakis, 2000). Revisões posteriores (Hall \& Taylor, 2003) reafirmavam o legado da Declaração de Alma-Ata. 20 anos depois, em maio de 1998, na sua 51a assembleia a World Health Organisation adoptou uma resolução corretiva da anterior para uma nova política de saúde global "The new policy, Health for All in the 21st Century", que sucedia à estratégia "Health for All by the Year 2000" lançada em 1977. Esta nova resolução visava lançar novo impulso para a saúde, e visava dominantemente o ano 2020 (WHA, 1998).

A World Health Organisation desenvolveu um programa para a região europeia, o Health21, dado a disparidade no desenvolvimento económico entre os países desta região e os países que eram abrangidos no programa anterior, na maioria países em desenvolvimento. Jogavam com o termo 21 porque propunham 21 objetivos para o início do século 21.

Nesta correção definiam 10 novos objetivos que se podiam operacionalizar de modo mais concreto e mensurável, com menor ambiguidade. Agrupavam-nos em três áreas: resultados de saúde com quatro objetivos; determinantes de saúde, com dois objetivos; políticas de saúde e sustentabilidade do sistema de saúde com quatro objetivos (van Herten \& van de Water, 1999).

\section{Promoção da saúde}

O conceito de promoção da saúde emerge na década posterior à referida antes e, formalmente, a nível planetário com a First International Conference on Health Promotion, uma reunião que teve lugar em Ottawa, Canadá, em 1986. Esta ocorre seis anos depois da reunião de Alma-Ata que estabeleceu os princípios para os CP a nível do planeta. Conferências posteriores tiveram lugar em Adelaide, Austrália, (1988), Sundsvall, Suécia (1991), e a quarta conferência a de Jakarta, Indonésia (1997) a primeira que teve lugar fora dos países desenvolvidos, seguidas da conferência em México City, México (2000), a sexta em Bangkok, Tailândia (2005), onde se atualiza a "Bangkok Charter for Health Promotion in a Globalized World", a sétima em Nairobi, Quénia (2009) e a oitava, regressa ao mundo desenvolvido em Helsínquia, Finlândia em $2013\left(^{3}\right)$.

Cada uma destas conferências atualiza o conceito de promoção da saúde e o que lhe está associado de educação para a saúde

\section{A educação para a saúde aplicada}

Todos os programas definidos pelas instituições internacionais, fossem a nível planetário fossem a nível regional (p.ex. Europa), assumiam a educação como instrumento central de intervenção. Abrangia contextos como a cidade, a escola, a comunidade, a empresa, as unidades de saúde, entre outras. Visava todos os grupos etários, das crianças pequenas aos idosos, com ou sem especificidades de género, em condições muito variadas, fosse promover ou proteger a saúde em grupos saudáveis, fosse melhorar a qualidade de vida em grupos com doenças crónicas, fosse prevenir as

\footnotetext{
${ }^{3}$ Ver relatórios das conferências em http://www.who.int/healthpromotion/conferences/en/
} 
doenças antes delas surgirem (prevenção primária) fosse em condições em que as doenças já estivessem instaladas (prevenção secundária e terciária).

Os programas educacionais são concebidos, por exemplo, para indivíduos com diabetes tipo 1, uma doença crónica que tende a surgir na infância e que acompanhará o indivíduo por toda a vida, exigindo medidas de higiene, tratamento e cuidados específicos. São programas diferentes dos programas de educação para a diabetes tipo 2 , que embora aborde a mesma doença, tem características diferentes da diabetes tipo 1, e tende a surgir em idades mais avançadas. Programas deste tipo devem ser desenvolvidos para um número imenso de doenças crónicas que tendem a acompanhar as pessoas na vida do dia a dia.

Como consequência da evolução nas possibilidades de diagnóstico e do tratamento assim como das condições de vida, as pessoas vivem cada vez mais tempo, e com doenças que há 50 anos eram fatais. Por exemplo, Rogers, et al. (1992) afirmam que no Medical Outcomes Study, um estudo longitudinal realizado em diferentes cidades dos Estados Unidos da América com uma população de 22462 indivíduos, dos participantes que tinham entre os 18 e 98 anos de idade, 3\% não apresentavam uma condição médica, 67\% apresentavam uma, e 30\% apresentavam duas ou mais condições. Ou seja, é hoje vulgar as pessoas da comunidade viverem a vida do dia a dia com uma doença. Essa coexistência da doença com a vida diária implica cuidados múltiplos, e tipos de intervenção onde a educação é uma parte importante. A educação deve ocorrer, portanto, com toda a população, ao longo de toda a vida.

As idades mais novas são intuitivamente mais focadas, porque as crianças estão em contexto escolar onde a intervenção educativa é mais acessível e onde os objetivos educativos contemplam áreas da saúde, normalmente associadas ao estilo de vida (ou life skills, para usar a linguagem da promoção da saúde). Os objetivos podem ser ligados à alimentação, ao exercício físico, ao consumo de substâncias como o tabaco, ao controlo da agressividade, à saúde sexual, entre muitos outros.

Em todas as idades, mais nas idades mais novas, temos que considerar o nível de desenvolvimento humano. O nível de desenvolvimento cognitivo deve ser considerado na formatação dos programas educativos (Pais-Ribeiro, 1996), e noutras idades deve ser considerado o nível de desenvolvimento intelectual, frequentemente associado ao nível de escolaridade, e às experiências de vida. Isto impõe no desenho, que programas que visam objetivos semelhantes (p.ex. promoção do exercício físico), sejam diferentes se se dirigem a crianças, a adultos jovens ou a idosos (com ou sem uma doença específica), dado que devem tomar em consideração o nível de desenvolvimento em que estão, as características culturais associadas à idade e experiência de vida, ou as características fisiológicas associadas à idade e condição do grupo.

A intervenção educativa pode ser realizada face a face, individualmente ou em grupo, ou indiretamente via diferentes media. As diferenças na intervenção dependem dos objetivos da intervenção, dos participantes e do contexto onde a intervenção ocorre. Inúmeros programas de intervenção indireta, utilizando diferentes media, têm sido desenvolvidos por todo o mundo, como na prevenção de doenças cardiovasculares, no Japão (Miyamatsu, et al., 2012), na Finlândia (Pietinen, et al. 1996), ou nos Estados Unidos da América (Fortmann, et al. 1995), na educação para a diabetes tipo 2 (Deakin, Cade, Williams, \& Greenwood, 2006), para a asma em crianças em contexto escolar a partir dos quatro anos de idade (Coffman, Cabana, \& Yelin, 2009), ou educação alimentar no pré escolar (D'agostino, D'andrea, Nix, \& Williams, 1999), entre muitos outros. Enfim, são amplos os estudos sobre a utilização de programas educativos, em 
todas as idades com variados objetivos, ligados à promoção, à proteção da saúde ou à prevenção de doenças. A sua importância e a sua utilidade estão demonstradas e são indiscutíveis.

A investigação mostra, exuberantemente, que os programas educativos são eficazes na mudança de comportamentos, de atitudes, ou de outras características psicológicas úteis para implementar estilos de vida associados a melhor saúde. Os profissionais e os contextos onde se realizam as intervenções devem estar atentos à investigação que mostra que os programas são eficazes mas que o objeto da intervenção não são os mesmos que têm estado em vigor nos últimos 60 anos como mostra, por exemplo, Ebrahimet al. (2011).

\section{REFERÊNCIAS}

Coffman, J.M., Cabana, M.D., \& Yelin, E,H. (2009).Do school-based asthma education programs improve self-management and health outcomes? Pediatrics, 124, 729742. doi: 10.1542/peds.2008-2085.

D'agostino, C., D'andrea, T., Nix, S., \& Williams, C. (1999) Increasing Nutrition Knowledge in Preschool Children: The Healthy Start Project, Year 1. Journal of Health Education, 30, 217-221. doi:10.1080/10556699.1999.10604642

Deakin, A., Cade, J., Williams, R., \& Greenwood, D.(2006). Structured patient education: the Diabetes X-PERT Programme makes a difference. Diabetic Medicine, 23, 944-954. doi: 10.1111/j.1464-5491.2006.01906.x

Declaration of Alma-Ata (1978). International Conference on Primary Health Care, Alma-ATA, USSR. Recuperado de http://www.who.int/publications/almaata_declaration_en.pdf

Ebrahim, S., Taylor, F., Ward, K., Beswick, A., Burke, M., \& Davey Smith, G. (2011). Multiple risk factor interventions for primary prevention of coronary heart disease. Cochrane Database of Systematic Reviews, 1. Art. No.: CD001561. doi: 10.1002/14651858.CD001561.pub3.

Fortmann, S., Flora, J., Winkleby, M., Schooler, C., Taylor, C., \& Farquhar, J. (1995). Community Intervention Trials: Reflections on the Stanford Five-City Project Experience. American Journal of Epidemiology, 142, 576-586.

Herrman, H., Saxena, S., \& Moodie, S. (edts.) (2005). Promoting mental health: concepts, emerging evidence, practice. Geneva, $\mathrm{CH}$ : World Health Organization.

Hall, J., \& Taylor, R. (2003). Health for all beyond 2000: the demise of the Alma-Ata Declaration and primary health care in developing countries. The Medical Journal of Australia, 178, 17-20.

Lalonde, M. (1974). A new perspective on the health of Canadians. Ottawa, CA: Minister of National Health and Welfare.

Miyamatsu, N., Kimura, K., Okamura, T., Iguchi, Y., Nakayama, H., Toyota, A.,...Yamaguchi, T. (2012). Effects of Public Education by Television on Knowledge of Early Stroke Symptoms Among a Japanese Population Aged 40 to 74 Years: A Controlled Study. Stroke, 43, 545-549. doi: 10.1161/STROKEAHA.111.634196

Pais-Ribeiro, J. (1996). Saúde e desenvolvimento. Análise psicológica, 16, 177-190.

Pais-Ribeiro, J. (1998). Psicologia e saúde. Lisboa, Portugal: ISPA. 
Pietinen, P., Vartiainen, E., Seppänen, R., Aro, A., \& Puska, P. (1996). Changes in diet in Finland from 1972 to 1992: impact on coronary heart disease risk. Preventive Medicine, 25, 243-250. doi: org/10.1006/pmed.1996.0053.

Richmond, J. (1979). Healthy people: the surgeon general's report on health promotion and disease prevention. Washington, D.C.: U. S. Department of Health, Education, and Welfare.

Ritsatakis, A. (2000). Experience in setting targets for health in Europe. European Journal of Public Health,10 (4 supp.), 7-10. doi:10.1093/eurpub/10.suppl_4.7

Rogers, W., McGlyn, E., Berry, S., Nelson, E., Perrin, E., Zubkoff, M.....Ware Jr, (1992). Methods of sampling. In: A. L. Stewart \& J. E. Ware Jr, (Edts). Measuring functional status and well-being (pp.27-48): The Medical Outcomes Study approach. Durham,NC: Duke University Press;

van Herten, L., \& van de Water, H. (1999). New global Health for All targets. British Medical Journal, 319, 700-703. doi:org/10.1136/bmj.319.7211.700

World Health Assembly. (1998). Health for all policy for the twenty first century. Geneva: World Health Organisation. (Resolution WHA51.7.).

World Health Organization (1998).Health Promotion Glossary. Geneve, CH: World Health Organization. (WHO/HPR/HEP/98.1).

World Health Organization (2013). Health Education. Geneva, CH: World Health Organization.

World Health Organization (1986). Young people's health- a challenge for society. World Health Organization (Technical Report Series,731). Geneve, CH: World Health Organization.

World Health Organization (1948).Officials Records of the World Health Organization, $\mathrm{n}^{\mathrm{o}}$ 2, p. 100. United Nations, World Health Organization, Interim Comission. Geneve, $\mathrm{CH}$ : World Health Organization. 